\title{
手性环丙烯构建策略用于对映选择性合成偕二氟亚甲基三元环化合物
}

\author{
欧阳瑶 $a$ 卿凤悀 $*, a, b$ \\ $\left({ }^{a}\right.$ 中国科学院上海有机化学研究所 有机氟化学重点实验室 上海 200032) \\ ( ${ }^{b}$ 东华大学化学化工与生物工程学院 生态纺织教育部重点实验室 上海 201620)
}

\section{New Strategy for the Construction of Chiral Cyclopropenes: Enantioselective Synthesis of Gem-difluoromethylenated Three-Membered Carbocycles}

\author{
Ouyang, Yao $^{a} \quad$ Qing, Fengling ${ }^{*}, a, b$ \\ ( ${ }^{a}$ Key Laboratory of Organofluorine Chemistry, Center for Excellence in Molecular Synthesis, Shanghai Institute of \\ Organic Chemistry, Chinese Academy of Sciences, Shanghai 200032) \\ $\left({ }^{b}\right.$ Key Laboratory of Science and Technology of Eco-Textiles, Ministry of Education, College of Chemistry, \\ Chemical Engineering and Biotechnology, Donghua University, Shanghai 201620)
}

作为最小的不饱和环状分子, 环丙烯独特的刚性结 构和多变的反应活性吸引了化学家的研究兴趣. 自 1922 年 Demjanov ${ }^{[1]}$ 报道了环丙烯化合物的首例合成以 来, 现已发展了一系列环丙烯的合成方法. 手性环丙烯 的合成是通过炔烃和重氮化合物的 $[2+1]$ 不对称环加成 反应. 根据底物的不同, 这些不对称环加成反应可以分 为四类: (a)末端炔烃和单取代重氮化合物的反应, (b)末 端炔烃和双取代重氮化合物的反应, (c)非末端炔烃和双 取代重氮化合物的反应, (d)非末端炔烃和单取代重氮化 合物的反应. 在这四类反应中, 末端炔烃和单取代重氮 化合物的不对称反应相对容易进行. 1992 年, Doyle 和 Müller 等 ${ }^{[2]}$ 报道了手性铑催化剂 $\left[\mathrm{Rh}_{2}(5 R \text {-MEPY })_{4}\right]$ 促进 的末端炔烃和重氮醋酸酯之间的不对称环丙烯基化反 应(Scheme 1a). 随后各种手性催化剂包括 $\left[\mathrm{Rh}_{2}(\mathrm{OAc})-\right.$ $\left.(\mathrm{DPTI})_{3}\right]^{[3]}$ 、 $\mathrm{Ir}(\mathrm{salen})$ 衍生 物 ${ }^{[4]}$ 和 $[\mathrm{Co}(3,5$-diMes-ChenPhyrin) $]^{[5]}$ 等被先后报道用于末端炔烃和单取代重氮化 合物的不对称 $[2+1]$ 环加成反应.

相对而言, 其它三类不对称 $[2+1]$ 环加成反应较为 困难. 2004 年, Davies 课题组 ${ }^{[6]}$ 报道了在手性铑催化剂 $\left[\mathrm{Rh}_{2}(S \text {-DOSP })_{4}\right]$ 作用下, 末端炔烃与芳基重氮醋酸酯的 对映选择性环丙烯化反应(Scheme 1b). 2012 年, Davies 小组 ${ }^{[7]}$ 又实现了在手性双金络合物 $[(S)$-xylylBINAP$(\mathrm{AuCl})_{2}$ ]催化下非末端炔烃和芳基重氮醋酸酯的不对称 环加成反应(Scheme 1c). 他们认为: 金催化相对于常用

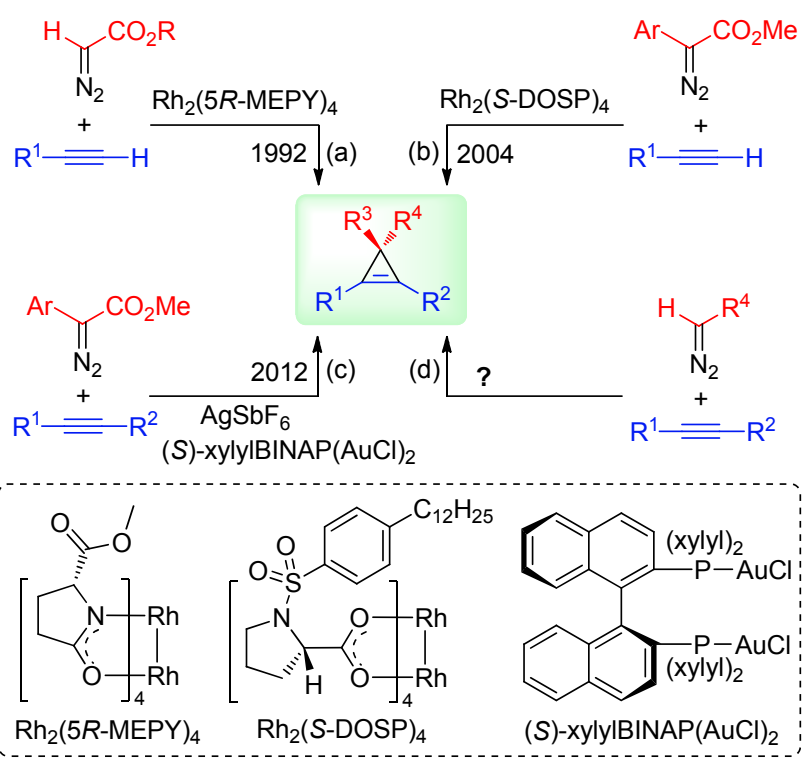

图式 1 炔烃和重氮化合物的不对称 $[2+1]$ 环加成反应

Scheme 1 Asymmetric [2+1] cycloaddition reactions of alkynes and diazo compounds

的铑催化而言，由于反应中间体金卡宾络合物对立体位 阻不那么敏感，因此能够高效地控制 donor/acceptor 卡 宾和位阻较大的末端炔烃反应的立体选择性. 令人遗憾 的是，非末端炔烃和单取代重氮化合物的不对称 $[2+1]$ 环加成反应一直没有报道(Scheme 1d).

最近天津大学理学院化学系马军安教授课题组报

* Corresponding author. E-mail: flq@mail.sioc.ac.cn. Published online March 3, 2020 
道了利用他们发展的二氟亚甲基砜基重氮化试剂 $\left(\mathrm{PhSO}_{2} \mathrm{CF}_{2} \mathrm{CHN}_{2}, \mathrm{Ps}-\mathrm{DFA}\right)^{[8]}$, 成功地实现了首例 $\mathrm{Rh}(\mathrm{II})$ 催化不对称非末端炔烃与单取代卡宾前体的环加成反 应(Scheme 2) ${ }^{[9]}$.

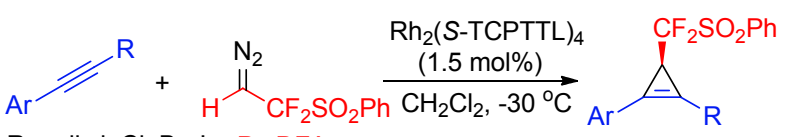

$\mathrm{R}=$ alkyl, $\mathrm{Cl}, \mathrm{Br}, \mathrm{I} \quad \mathrm{PS}-\mathrm{DFA} \quad 32$ examples up to $97 \%$ yield, $97 \%$ ee

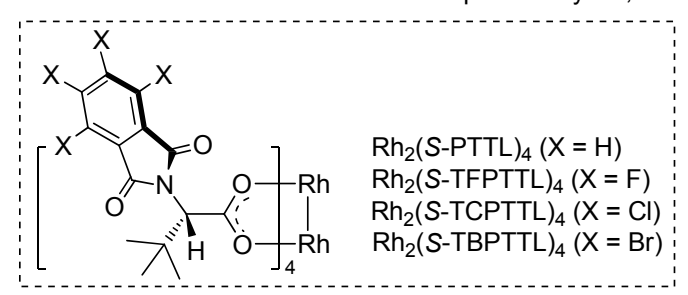

图式 $2 \mathrm{Rh}(\mathrm{II})$ 催化的对映选择性的非末端炔烃和 Ps-DFA 的 环丙烯化反应(催化剂量为 $1.5 \mathrm{~mol} \%$ )

Scheme $2 \mathrm{Rh}^{\mathrm{II}}$-catalyzed enantioselective cyclopropenation reaction of internal alkynes and Ps-DFA

他们发现在较低的温度下 $\mathrm{Du}$ Bois 催化剂 $\left[\mathrm{Rh}_{2} \mathrm{esp}_{2}\right]$ 可以催化非末端炔烃和 Ps-DFA 的反应, 以中等产率得 到目标产物. 他们进一步篎选不同的手性 $\mathrm{Rh}(\mathrm{II})$ 催化剂, 包括 $\mathrm{Rh}_{2}(S \text {-DOSP })_{4}, \mathrm{Rh}_{2}(\mathrm{OAc})(\mathrm{DPTI})_{3}$ 和 $\mathrm{Rh}_{2}(S \text {-PTTL })_{4}$. 其中 $\mathrm{Rh}_{2}(S-\mathrm{PTTL})_{4}{ }^{[10]}$ 可以得到较高的产率和 $e e$ 值. 最后 通过对配体的优化, 发现 $\mathrm{Rh}_{2}(S \text {-TCPTTL })_{4}$ 可以显著地 提高 $e e$ 值. 在最优反应条件下, 一系列非末端炔烃均能 以较高的收率和 $e e$ 值得到对映体富集的偕二氟亚甲基 砜基取代的环丙烯化合物. 该反应具有广泛的底物普适 性和官能团兼容性. 例如苯环上取代的强吸电子基: $\mathrm{CN} 、 \mathrm{CF}_{3}$ 和 $\mathrm{NO}_{2}$ 均能兼容. 萗环、噻吩环以及吡啶环取 代的炔烃底物也同样适用.

该反应得到的环丙烯产物能够发生脱砜化、氢化还 原、Diels-Alder 反应以及 Pauson-Khand 反应等, 这些转 化反应具有高度的立体选择性, 通过这些转化反应可得 到在合成上非常有用的手性含氟合成砌块(Scheme 3).

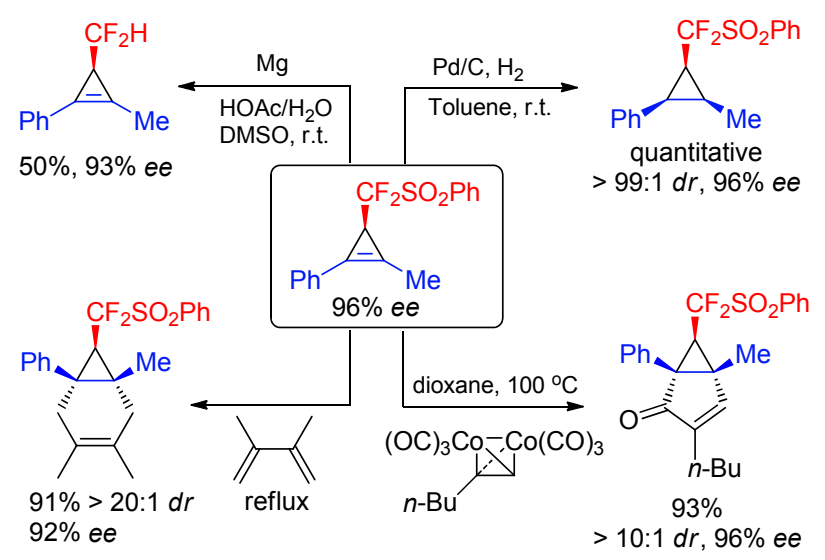

图式 3 偕二氟亚甲基化环丙烯的合成转化

Scheme 3 Synthetic transformation of gem-difluoromethylenated cyclopropene

总之，马军安教授课题组的工作解决了非末端炔烃 和单取代卡宾前体的对映选择性环加成问题，丰富了构 建手性环丙烯的合成方法学. 但还有一些问题尚待研 究，例如三氟甲基取代的卡宾前体与末端炔烃以及非末 端炔烃的不对称环加成反应.

\section{References}

[1] Demjanov, N. Y.; Doyarenko. M. N. Bull. Acad. Sci. Russ. 1922, 16, 297.

[2] Protopopova, M. N.; Doyle, M. P.; Müller, P.; Ene, D. J. Am. Chem. Soc. 1992, 114, 2755.

[3] Lou, Y.; Horikawa, M.; Kloster, R. A.; Hawryluk, N. A.; Corey, E. J. J. Am. Chem. Soc. 2004, 126, 8916.

[4] Uehara, M.; Suematsu, H.; Yasutomi, Y.; Katsuki, T. J. Am. Chem. Soc. 2011, 133, 170.

[5] Cui, X.; Xu, X.; Lu, H.; Zhu, S.; Wojtas, L.; Zhang, X. P. J. Am. Chem. Soc. 2011, 133, 3304.

[6] Davies, H. M. L.; Lee, G. H. Org. Lett. 2004, 6, 1233.

[7] Briones, J. F.; Davies, H. M. L. J. Am. Chem. Soc. 2012, 134, 11916.

[8] Zeng, J.-L.; Chen, Z.; Zhang, F.-G.; Ma, J.-A. Org. Lett. 2018, 20, 4562 .

[9] Zhang, Z. -Q.; Zheng, M.-M.; Xue, X.-S., Marek, I.; Zhang, F.-G.; Ma, J.-A. Angew. Chem., Int. Ed. 2019, 58, 18191.

[10] Kitagaki, S.; Anada, M.; Kataoka, O.; Matsuno, K.; Umeda, C.; Watanabe, N.; Hashimoto, S. J. Am. Chem. Soc. 1999, 121, 1417.

(Lu, Y.) 